\title{
The Prevalence of diabetic optic neuropathy in type 2 diabetes mellitus
}

Ali A. Taqi Al-Saffar*

\section{Abstract}

Background and objective: As diabetes mellitus a common health problem, it is well known that it can lead to optic neuropathy that affects the optic nerve functions. It is important to monitor the effect of this metabolic disease on the optic nerve that can lead ultimately to decrease visual acuity that can be irreversible. This study aimed to find out the prevalence of diabetic optic nerve diseases and to evaluate the patient characteristics and fundus findings.

Methods: Screening examination was done for 2213 patients with type 2 diabetic patients presented to the diabetic center from October 2007 to September 2009. The examination includes visual acuity test using conventional $E$ chart, slit lamp exam, followed by installing short acting Mydriatics (tropicamide 1\%) eye drops for fundoscopy examination using +76.D or +90 D.

Results: Eighty eight patients (approximately 4\%) had optic nerve problems; 50 females and 38 males. The mean age was 59 years. A total of 58 (116 eyes) patients were bilaterally affected, 42 patients with optic papillopathy, 8 patients with anterior ischemic optic neuropathy and profound loss of vision, 8 with glaucomatous cupping and pallor and 30 patients with end stage optic atrophy. A total of $63(71.5 \%)$ patients had poor metabolic control.

Conclusions: Patients with type 2 diabetes mellitus have $4 \%$ prevalence of diabetic optic neuropathy.

Keywords: Background diabetic retinopathy; Proliferative diabetic retinopathy; Primary open angle glaucoma; Anterior ischemic optic neuropathy.

\section{Introduction}

Diabetic papillopathy is an atypical form of non-arteritic anterior ischemic optic neuropathy (NAION). Patients with juvenile diabetes, transient unilateral or bilateral optic disc edema often develops with mild or absent visual symptoms. Blind spot enlargement is more commonly seen than an arcuate field defect. Although these field defects may persist, visual acuity usually improves as the disc edema resolves. The dilated, telangiectatic vessels on the disc, mimicking neovascularization, disappear as disc edema resolves. The formation of these vessels is thought to be related to the luxury perfusion phenomenon described after typical NAION. Diabetic papillopathy may develop in patients with or without diabetic retinopathy. Diabetic papillopathy may also be seen in patients with adult-onset diabetes mellitus. ${ }^{1}$ Acute optic disc edema associated with diabetes, or diabetic papillopathy, usually occurs in the second to fourth decades of life and generally shows no correlation with the severity of diabetic retinopathy. It is typically associated with mild loss of vision $(\geq 20 / 50)$ and the visual field may be normal or may show defects, such as an increased blind spot, arcuate scotomas, or altitudinal scotomas. ${ }^{2}$ Diabetic papillopathy differs from anterior ischemic optic neuropathy (AION). Typical AION is generally seen in middle-aged to elderly frequently hypertensive persons with or without diabetes, and is characterized by acute

* Sulaimani School of Medicine, Sulaimani, Iraq. 
unilateral moderate-to-marked loss of vision, swelling of the optic disc with variable nerve fiber layer hemorrhages, segmental areas of non-perfusion on fluorescein angiography, poor prognosis for visual recovery, and late optic disc pallor. ${ }^{2}$ Diabetic papillopathy (DP) is a term that is used to characterize a finding of unilateral or bilateral optic disc edema with variable visual loss in a patient with diabetes. Specific criteria for the diagnosis of diabetic papillopathy and a clear differentiation of this entity from Non-arteritic anterior ischemic optic neuropathy (NAION) have not been established. While diabetic papillopathy has a generally favorable prognosis and does not usually require treatment, it must be distinguished from more malignant processes such as papilledema secondary to increased intracranial pressure. ${ }^{3}$ As diabetes mellitus is one of the most common metabolic diseases and it causes significant morbidity to body organs leading to damage and faulty function, the eye and optic nerve can suffer problems termed as diabetic optic neuropathy and one of the problems that is still underestimated as it is clinically subtle is the diabetic papillopathy that if recur can lead to significant visual loss. This study aimed to find out the prevalence of diabetic optic nerve diseases and evaluate the patient characteristics and fundus findings.

\section{Methods}

Patients enrolled are diabetic type II patients presented to the diabetic center in Sulaimaniya city, Kurdistan region, Iraq. Started from October 2007 to September 2009, in organized file and computer documentation, first examining visual acuity by conventional Snellen $E$ chart, dilated fundoscopy done (using $+90 \mathrm{D}$ or $+76 \mathrm{D}$ with the aid of slit lamp) after about 30 minutes from short acting mydriatics tropicamide installed in the eyes to be examined (unless the patient having shallow AC), 2213 patients examined. The following are brief definitions of different optic nerve diseases:

Diabetic papillopathy depends on clinical examination visual acuity which is mildly affected with Fundoscopy that shows variable disc hyperemia and pallor. ${ }^{1}$

Non-arteritic anterior ischemic optic neuropathy (NAION) is characterized by the following triad: abrupt (usually monocular), painless loss of vision; optic disc swelling with or without surface hemorrhages and cotton-wool spots; and nerve fiber bundle defects in the visual field of the affected eye. ${ }^{4}$

Primary open angle glaucoma (POAG) also known as chronic simple glaucoma of adult onset and is typically characterized by slowly progressive raised intraocular pressure $(>21 \mathrm{mmHg}$ recorded on at least a few occasions) associated with characteristic optic disc cupping and specific visual field defects. ${ }^{5}$

Optic atrophy is the final common result of injury to the retinal ganglion cells, nerve fiber layer, optic nerve, chiasm, or optic tract. The clinical hallmark of non-glaucomatous optic atrophy is pallor of the optic disc, seen ophthalmoscopically. ${ }^{6}$ Regarding visual acuity loss it ranges from: Normal vision extends beyond 1.0 (20/20, $6 / 6$ ). It is important to realize that the visual acuity value of $1.0(20 / 20,6 / 6)$.

Mild Loss Special attention needs to be given to the second range. This is a transitional range between fully normal vision and Low Vision. Many states set their driver's license requirement at 0.5 $(20 / 40,6 / 12)$. In the U.S. special education assistance becomes available at $<0.3$ $(<20 / 60,<6 / 18)$. The range may then be subdivided into "minimal loss" (0.63 and 0.5 ) and "mild loss" (0.4 and 0.32).

Profound Vision Loss ( $<0.05$, < 20/400, $<3 / 60)^{7}$

About metabolic control: Average of serial checks of blood sugar with the checking of $\mathrm{HbA} 1 \mathrm{c}$ according the chart.

Inclusion Criteria: Type 2 non-insulin dependent diabetic patients with good to fairly clear fundus view.

Exclusion Criteria: Patient with type 1 
Zanco J. Med. Sci., Vol. 21, No. (3), December, 2017 https://doi.org/10.15218/zjms.2017.051

diabetes mellitus, wolfram syndrome (DIDMOAD) very hazy unclear fundus view, patients refusing dilated Fundoscopy and patients with shallow anterior chamber angle.

Statistical analysis: Microsoft excels 2010, Chi square and $P$ value.

Ethical committee approval: Order No: 81-08, date of approval 19/4/2008 (copy included).

\section{Results \\ Out of 2213 patients examined,}

88 (146 eyes) patients suffered optic neuropathy, which represents approximately $4 \%$ of cases, as shown in Figure1. Regarding the demographic characteristics of patients summarized in Table 1. Females represented $56.2 \%$ of cases and males $43.8 \%$. The age range was $39-85$ years and the average age was about 58.9 years. The late middleaged group represented about 55\% of cases. Bilateral neuropath was present in $58(65.9 \%)$ patients.

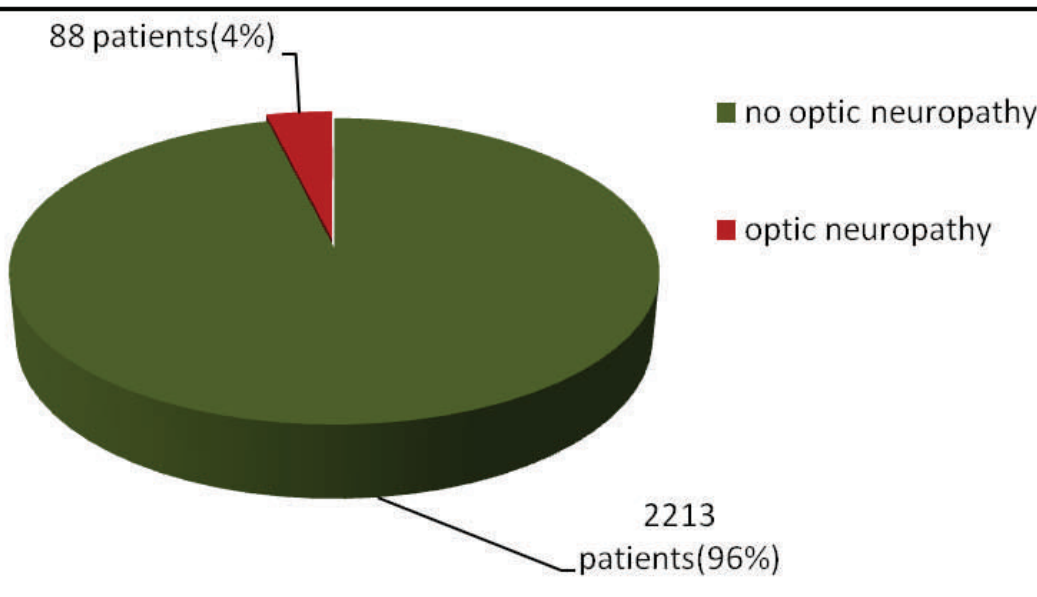

Figure 1: The prevalence of diabetic optic neuropathy.

Table1: Demographic characteristics of the involved patients.

\begin{tabular}{lcc}
\hline Variable & Number & Percentage (\%) \\
\hline Gender & 38 & $(43)$ \\
male & 50 & $(57)$ \\
female & & \\
Laterality & 30 & $(34)$ \\
Unilateral & 58 & $(66)$ \\
Bilateral & & $(16)$ \\
Age groups & 14 & $(55)$ \\
$39-49$ & 49 & $(29)$ \\
$50-64$ & 25 & $(63)$ \\
$65-84$ & 56 & $(37)$ \\
Duration of diabetes & 32 & $(28)$ \\
Less than 10 years & & $(72)$ \\
Equal or more than 10 years & 25 & \\
Patients social status & 63 & $(100)$ \\
Employees and workers & & \\
Retired and housewives & 88 & \\
Total & & \\
\hline
\end{tabular}


Zanco J. Med. Sci., Vol. 21, No. (3), December, 2017 https://doi.org/10.15218/zjms.2017.051

Of diabetes duration up to 10 years, we had the majority that represented $63 \%$ of cases and they had no significant diabetic retinopathy. A total of $25(28 \%)$ patients were in there active working time that may affect their productivity and 63 (72\%) patients needed to be taken care more by educating of them and their close relatives. Most of affected patients (72\%) were retired and housewives while $28 \%$ were active working patients. Optic nerve diseases presented in
Different pathologies as summarized in Figure 2. The majority presented with diabetic papillopathy and good percentage unfortunately with optic atrophy. Regarding the associated diabetic retinopathy, the majority $(50 \%)$ of patients had no significant retinopathy, 33 (37\%) patients had various degrees of background diabetic retinopathy while 11 $(13 \%)$ patients has proliferative diabetic retinopathy as shown in Figure 3.

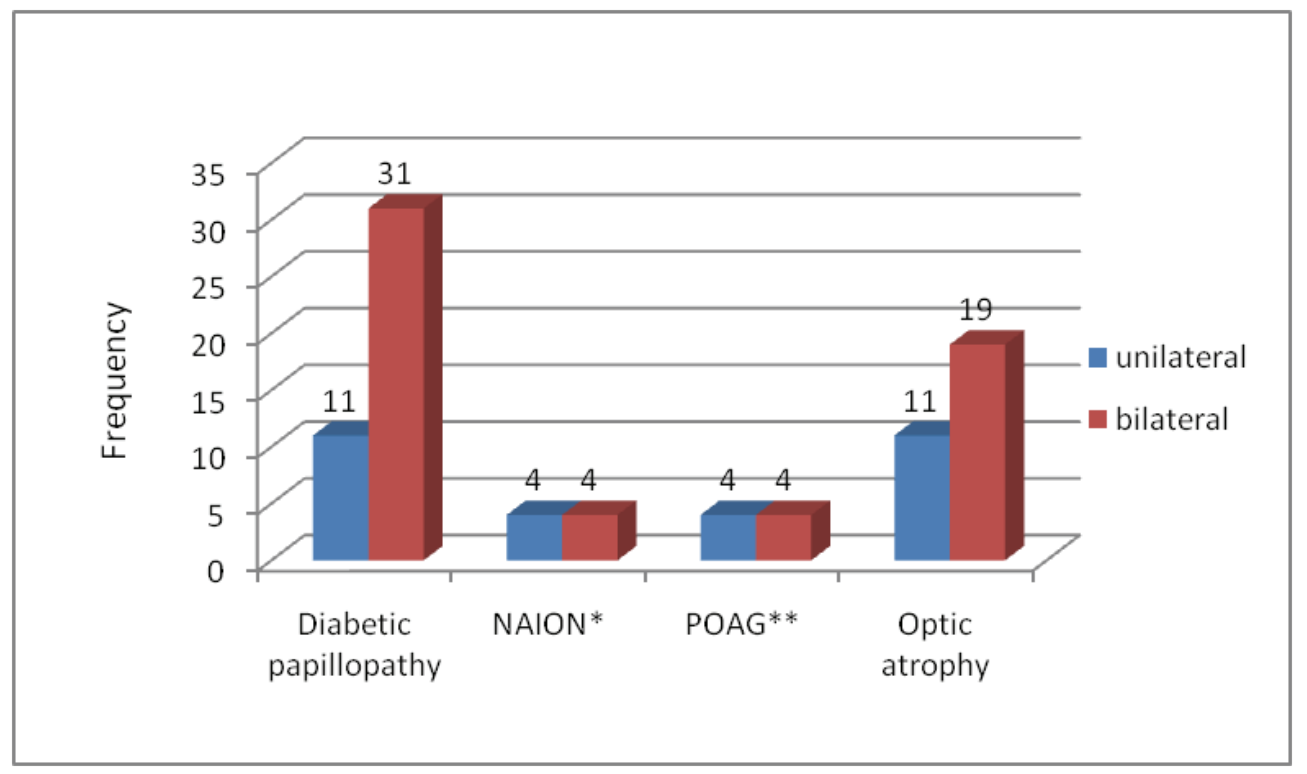

Figure 2: Frequency of each type of optic nerve disease.

${ }^{*}$ None-Arteritic Anterior ischemic optic neuropathy. ${ }^{* *}$ POAG: Primary open angle glaucoma.

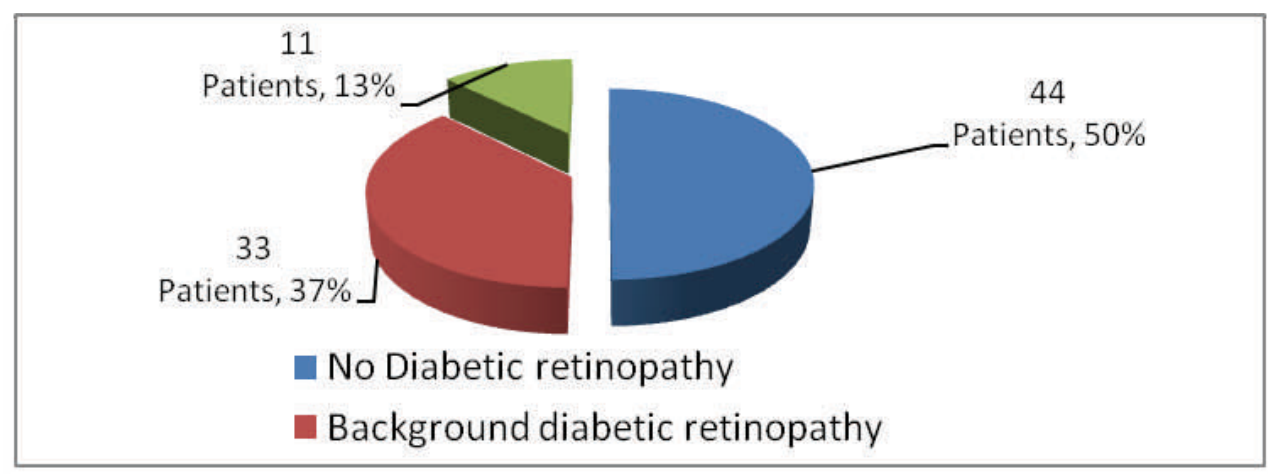

Figure 3: The association between optic neuropathy and diabetic retinopathy. 
Zanco J. Med. Sci., Vol. 21, No. (3), December, 2017

https://doi.org/10.15218/zjms.2017.051

Of 11 (18 eyes) patients having PDR; 9 eyes PRP done and the other 9 eyes need to be done. Regarding treatment, 25 $(28.4 \%)$ patients had good metabolic control, while $63(72.6 \%)$ patients were metabolically not well controlled (Table 2). Visual acuity ranged from normal, minimum, mild to profound visual loss and legally blind eyes that clinically associated with optic nerve diseases. Visual acuity results, normal to mild effect in $69(39.6 \%)$ eyes, mild to moderately affected in 62 $(35.6 \%)$ eyes and moderately to profound (severe) affected in $43(24.7 \%)$ eyes (Figure 4).

Table 2: The treatment modes and the metabolic control pattern.

\begin{tabular}{lccccc}
\hline \multirow{2}{*}{ Treatment } & $\begin{array}{c}\text { Oral hypoglycemic } \\
\text { No. (\%) }\end{array}$ & $\begin{array}{c}\text { Insulin and Oral } \\
\text { No. (\%) }\end{array}$ & $\begin{array}{c}\text { Insulin only } \\
\text { No. (\%) }\end{array}$ & No & P value \\
\hline Well control* $^{*}$ & $21(30.8)$ & $2(15.3)$ & $2(28.5)$ & 25 & \\
Uncontrolled $^{* *}$ & $47(69.1)$ & $11(84.6)$ & $5(71.4)$ & 63 & 0.731 \\
Total No. & 68 & 13 & 7 & 88 & \\
\hline
\end{tabular}

*well controlled: Mean blood sugar less or equal $180 \mathrm{mg} / \mathrm{dl}$ and HbA1c less or equal 8.0 according the diabetic control chart. ${ }^{5}$

${ }^{* *}$ uncontrolled: Abnormal mean blood sugar more than $180 \mathrm{mg} / \mathrm{dl}$ and HbA1c more than 8.0. ${ }^{5}$

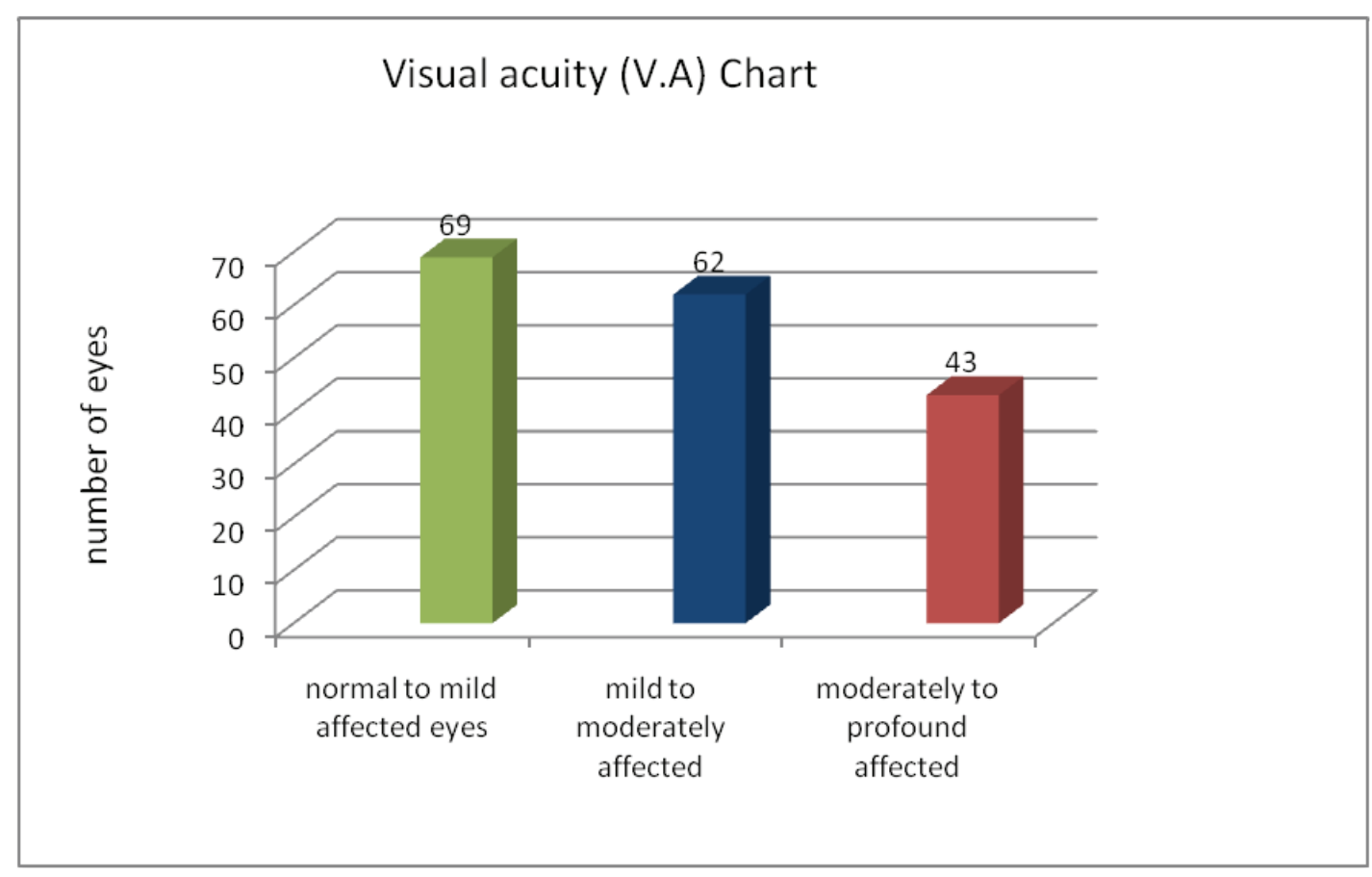

Figure 4: Visual acuity loss. 


\section{Discussion}

When we compare the results of this study with other studies, it is found in a study done in outpatient Ophthalmology Clinic in Nis-France; Out of 600 patients with diabetes, 314 were male and 286 female, with average age structure of 61.2 years. 467 patients were on oral anti-diabetic therapy, while 133 were on insulin therapy. Out of this number, there were 14 patients $(2.4 \%)$ with optic nerve disease. ${ }^{8}$ While in this study, the prevalence was higher and it is $4 \%$, the female $(57 \%)$, males $(43 \%)$, nearly comparable although we have the female more than the male number, a slightly less average age 58.50 years, our patients that having the optic nerve diseases are on oral hypoglycemic drugs $(77 \%)$, on insulin only( $8 \%)$ and on combined therapy(15\%). In comparison to a study done in Turkey; Twenty-four eyes of 16 patients with type II diabetes mellitus met the criteria, patients had a mean age of $57.1(+/-8.8)$ and approximately half of the patients had poor metabolic control. Disk swelling was bilateral in $8(50 \%)$. $13(54 \%)$ eyes had non-proliferative and $2(8 \%)$ eyes had proliferative diabetic retinopathy at presentation. ${ }^{9}$ In this study overall prevalence of diabetic papillopathy present in $42(1.89 \%)$ patients, it represents $47.7 \%$ from overall 88 patients with optic neuropathy, average years, poor metabolic control $63 \%$ of patients, bilateral cases present in $58(65.9 \%)$ patients, and 35 (24\%) eyes with DRP and $18(12.3 \%)$ eyes with PDR (proliferative diabetic retinopathy) respectively. In another study, Twentyseven eyes of 19 patients met the study definition of diabetic papillopathy. Patients were generally older (mean age, 50 years) and of a broader age range (19 to 79 years) compared with those in prior reports. Two thirds of patients had type II diabetes. ${ }^{10}$ In a study of 19 patients with diabetic papillopathy, 69 the mean age of onset was 50 years of age and $33 \%$ had type II diabetes mellitus. Hyperemic disc edema resolved within 3.7 months. Seventy percent of eyes had macular edema and
$52 \%$ of eyes had capillary non-perfusion on fluorescein angiography. Eighty-nine percent (34 of 38) of eyes had a final visual acuity of $20 / 50$ or better. ${ }^{11}$ The condition presents bilaterally in approximately $50 \%$ of cases. The visual prognosis is usually good, with nearly all younger patients recovering to a visual acuity of $\geq 20 / 30$ (6/9). In diabetic papillopathy, diffuse disc swelling may mimic papilledema of raised intracranial pressure. To avoid unnecessary PRP (Panretinal photocoagulation), it is important to differentiate the prominent telangiectasia of disc vessels often seen in diabetic papillopathy from neovascularization of the disc. $^{2}$ While in this work the visual acuity get normal or mildly affected in $39.6 \%$, moderately affected in $35.6 \%$ and severely affected in $24.7 \%$ respectively, so it is about $75.2 \%$ of eyes have good or useful vision and about $24.7 \%$ of eyes get compromised vision and legally blind eye. In a study of 19 patients with diabetic papillopathy, 69 the mean age of onset was 50 years of age and $33 \%$ had type II diabetes mellitus. Hyperemic disc edema resolved within 3.7 months. Seventy percent of eyes had macular edema and $52 \%$ of eyes had capillary non-perfusion on fluorescein angiography. Eighty-nine percent (34 of 38) of eyes had a final visual acuity of $20 / 50$ or better. ${ }^{1}$ Non-arteritic anterior ischemia optic neuropathy (NAION) is a multifactorial disease in which some systemic diseases may act as predisposing factors and others as precipitating factors. Patients with anterior ischemia optic neuropathy show no significant increase in mortality, but those with both arterial hypertension and diabetes mellitus have significantly $(\mathrm{P}<.01)$ increased incidence of cerebrovascular disease. ${ }^{13}$ This study reveals that $8(9 \%)$ patients have NAION and all of them having associated badly control diabetes and hypertension. An increased risk of NAION occurs in $47 \%$ of patients with hypertension and $24 \%$ of patients with diabetes. Diabetes, 
hypertension, and hypercholesterolemia are more associated with NAION in younger patients less than 50 years of age than in older patients. ${ }^{1}$ It is noticed in our patients that diabetic optic neuropathy not related to the severity of diabetic retinopathy as we have $50 \%$ of cases not associated with significant diabetic retinopathy, Also it is noticed in one study that optic disk edema does not correlate with the degree of diabetic retinopathy. ${ }^{12}$ More reports that are recent suggest that older patients and patients with type 2 diabetes may actually comprise the majority of cases. A history of poor metabolic control present in $50 \%$ of patients. ${ }^{1}$ In our patients the poor metabolic control represents $63(71 \%)$ patients which are comparable to this finding.

\section{Conclusion}

A significant percentage (4\%) of diabetics type II had optic nerve problems with variable effect on visual acuity. It represents large number of patients as diabetes mellitus affects millions of population and it is increasing rapidly with time. Diabetic optic nerve diseases carry significant insult on visual abilities and it is more common in poor metabolic control.

\section{Conflicts of interest}

The author reports no conflicts of interest.

\section{References}

1. Chan JW. Ischemic optic neuropathy. In: Jane W Chan, editor. Optic Nerve Disorders Diagnosis and Management, $1^{\text {st }}$ ed. University of Kentucky College of Medicine, Lexington, Kentucky: Springer publishing; 2007. p. 34-37.

2. Ingrid US, Harry WF. Non-retinal Ocular Abnormalities. In: Ingrid US, Harry WFJr, William ES, editors. Diabetes and Ocular Disease Past, Present, and Future Therapies, $2^{\text {nd }}$ Edition. New York: Oxford University Press in cooperation with the American Academy of Ophthalmology; 2010. p.64 and 328

3. Madhura T, Nicholas J. Diabetic papillopathy-Up to Date; 2016. (Accessed November 29, 2016). Available at: http://www.uptodate.com/ contents/ diabetic papillopathy? source= search_result\&search=diabetic+papillopathy\&sele ctedTitle $=1 \sim 2$
4. Helmut W, Ulrich S. Optic Disc Signs and Optic Neuropathies. In: Ulrich S, Helmut W, William $\mathrm{Ha}$, Editors. Clinical Neuro- Ophthalmology a Practical Guide. $1^{\text {st }}$ ed. Berlin: Springer Verlag; 2007. p.118.

5. Khurana AK. Glaucoma.In: A K Khurana, editor. Comprehensive Ophthalmology, $4^{\text {th }}$ ed.New Delhi: New Age International (P) Limited, Publishers; 2007. p.214.

6. Nathan $T$, Randy $H$. Optic atrophy. In: Leonard A, Daniel M, editors. Ocular Disease: Mechanisms and Management, $1^{\text {st }}$ ed. New York: Elsevier Publishing; 2010. p. 339.

7. Visual Standards Report Prepared For the International Council of Ophthalmology at the 29th International Congress of Ophthalmology, Sydney, Australia April; 2002. (Accessed November 29, 2016, at http://www.icoph.org/ downloads/visualstandardsreport.pdf

8. Dragan V, Milo J. Diabetes mellitus and optic nerve diseases. ActaFac Med Naiss 2005; 22(3):145-8.

9. Bayraktar Z, Alacali N, Bayraktar S. Diabetic papillopathy in type II diabetic patients. Retina 2002; 22(6):752-8.

10. Regillo CD. Diabetic papillopathy. Patient characteristics and fundus findings; Arch Ophthalmol I995; 113(7):889-95.

11. David JB.Optic Nerve Disease in Diabetes Mellitus. David JB editor. Diabetic Retinopathy Evidence-Based Management. $1^{\text {st }}$ ed. New York, USA: Springer; 2010. p 361.

12. Hayreh SS1, Joos KM, Podhajsky PA. Long. Systemic Diseases Associated with Nonarteritic Anterior Ischemic Optic Neuropathy. Am J Ophthalmol 1994; 118(6):766-80. 